\title{
Integrated Parcellation and Normalization Using DTI Fasciculography
}

\author{
Hon Pong $\mathrm{Ho}^{1}$, Fei Wang ${ }^{4}$, Xenophon Papademetris ${ }^{1,3}$, Hilary P. Blumberg ${ }^{3,4}$, \\ and Lawrence H. Staib ${ }^{1,2,3}$ \\ ${ }^{1}$ Departments of Biomedical Engineering \\ ${ }^{2}$ Departments of Electrical Engineering \\ ${ }^{3}$ Departments of Diagnostic Radiology \\ ${ }^{4}$ Departments of Psychiatry, \\ Yale University, New Haven, CT, USA
}

\begin{abstract}
Existing methods for fiber tracking, interactive bundling and editing from Diffusion Magnetic Resonance Images (DMRI) reconstruct white matter fascicles using groups of virtual pathways. Classical numerical fibers suffer from image noise and cumulative tracking errors. $3 \mathrm{D}$ visualization of bundles of fibers reveals structural connectivity of the brain; however, extensive human intervention, tracking variations and errors in fiber sampling make quantitative fascicle comparison difficult. To simplify the process and offer standardized white matter samples for analysis, we propose a new integrated fascicle parcellation and normalization method that combines a generic parametrized volumetric tract model with orientation information from diffusion images. The new technique offers a tract-derived spatial parameter for each voxel within the model. Cross-subject statistics of tract data can be compared easily based on these parameters. Our implementation demonstrated interactive speed and is available to the public in a packaged application.
\end{abstract}

\section{Introduction}

Local white matter fiber orientation can be estimated from a set of diffusion weighted images (DWI) [1] by fitting to a diffusion distribution model. The classical second order diffusion model is represented using an ellipsoid, which is mathematically denoted as a symmetric positive definite tensor. The 3D field of ellipsoids becomes the Diffusion Tensor Image (DTI) [2]. Since neuronal fiber diameter is much smaller than a voxel, the measurements represent averaged orientations over a small region 3 . With an end goal of understanding and characterizing brain tracts in vivo, existing white matter parcellation methods from diffusion images can be summarized by two main steps: tracking and bundling.

\section{Existing Fiber Tracking and Bundling Paradigm}

Streamline tracking is the process of recovering line structures in a vector field 45:6]. The vector field $\mathbf{F}(\mathbf{x})$ is provided by orientations from DTI or either 

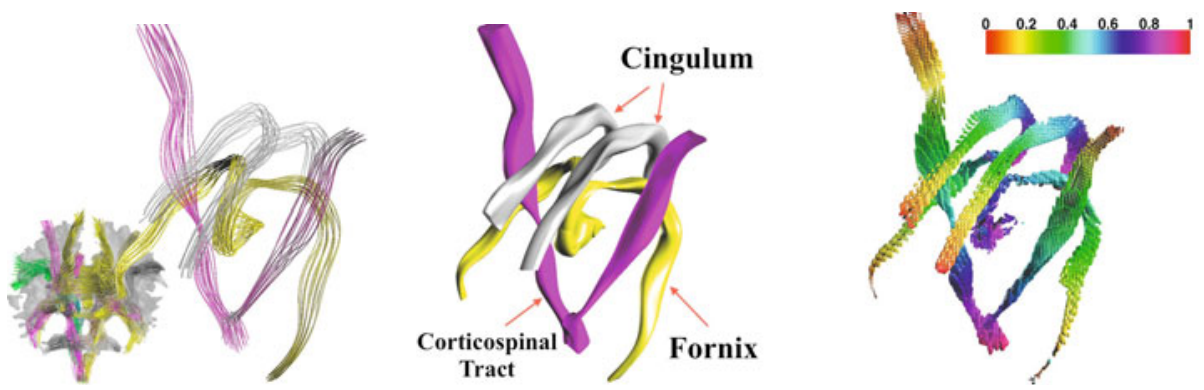

Fig. 1. Fasciculography of major white matter tracts from a human brain DTI. Fasciculography offers both the fibers view (left) and the tract view (middle) without additional processing. All tracts are intrinsically normalized, parametrized (right) and ready for cross-subject analysis.

orientation distribution models. The tracking problem can be written as an initial value problem of the partial differential equation [7]:

$$
\frac{d L(s)}{d s}=\mathbf{F}(L(s)) \quad, L\left(s_{0}\right)=\mathbf{x}_{0}
$$

where $s \in \mathbb{R}$ is the arc-length parameter of the streamline $L(s): \mathbb{R} \rightarrow \mathbb{R}^{3}$ in the $3 \mathrm{D}$ vector field $\mathbf{F}(\mathbf{x}): \mathbb{R}^{3} \rightarrow \mathbb{R}^{3}$, where $\mathbf{x} \in \mathbb{R}^{3}$ is a $3 \mathrm{D}$ point in the DTI. The best choice of seed points varies across tract types and subjects, and is critical for accurate tract segmentation.

A specific brain tract can be extracted and visualized by properly seeding, tracking and processing those virtual fiber pathways. The selection process has been performed manually or automatically using pattern recognition algorithms (classification/clustering). Interactive parcellation [89 usually involves manual post-editing of tracking results, either using whole brain seeding or individual region of interest (ROI) seeding. Automatic bundling [1011,12 can be done with the help of proper prior models. However, the availability and appropriateness of a training set poses limitations to methods using prior models. Most recently, a generalized cylinder approach has been proposed [13], but their tract solutions are limited to circular cross-sectional areas which is typically not a valid assumption for neural tracts. Moreover, their approach is a tubular-constrained level set surface evolution where the parameterization within the tract is not carefully considered and therefore tract normalization is still an issue.

Cross-subject analysis of fiber tracts is also hampered by the definition of point correspondence among bundles with varying fiber lengths and number of fibers. Various Tract-based Morphometry (TBM) methods [14 15]12] tackle the point correspondence issue mainly by generating a common parametrization through post-processing and registering fibers. However, tract parcellation based on fiber bundling relies on good fiber tracking results, while local orientation error from diffusion signals will significantly alter their trajectories. 
Motivation of the New Volumetric Approach: Our idea is a fast method that performs streamline tracking and anatomical segmentation simultaneously using a parametric volumetric representation, with less dependence on seed point locations and local orientation errors. Once a tract volume is delineated, we label each image voxel according to the axial parameters $\alpha \in[0, \pi]$ (similar to the arc-length parameter of a curve) of the tubular model. Then, the statistics of the tract image data can be computed based on those labels. For example, we can compare data of the anterior portion (first 20\%) of multiple fascicles using $\alpha \in[0-0.2 \pi]$. In [16], we proposed a fast volumetric tract extraction method using a pair of seed points. We here significantly reduce the dependancy of the seed pairs to one seed and tackle more complex tract geometry by a tract optimization algorithm.

\section{Methodology}

Our goal is to find a parametric volume $V(\alpha, \beta, \gamma): \mathbb{R}^{3} \rightarrow \mathbb{R}^{3}$ where $\alpha, \beta, \gamma \in$ $[0,2 \pi]$ and $\alpha, \gamma$ are symmetric, which best represents an oriented structure in a vector field. We intend to maximize the length of the tract axis through iterative filtering (Step 3) and streamline extension (Step 5) of a tract volume. Fibers for visualization can be generated by sampling the final $V(\alpha, \beta, \gamma)$ along the axial direction. Throughout this section, $\alpha, \beta$ and $\gamma$ refer to the axial, circumferential and radial parameters of a tract volume, respectively.

\subsection{Cross-Sectional Tractness Measure}

We would like to quantify how relevant a nearby voxel $\mathbf{x} \in \mathbb{R}^{3}$ is to a streamline $L(s)$ based on its orientation. The coherence of an oriented voxel given a streamline is defined as the inner product of the tensor orientation and the normalized tangent of the given streamline [16]:

$$
C(\mathbf{x} ; L(s))=\left(F(\mathbf{x}) \cdot \frac{d L(s)}{d s}\right)\left\|\frac{d L(s)}{d s}\right\|^{-1}
$$

Note that this coherence measurement is dependent on the streamline tangent at $s$. We cannot simply apply a threshold or perform region growing on this measurement without knowing the streamline orientation at $s$ for each $\mathrm{x}$.

Let $P_{Y(\alpha)}(\mathbf{p}) \in \mathbb{R}^{2} \rightarrow \mathbb{R}^{3}$ be the orthogonal plane of streamline $Y(\alpha)$, where $\mathbf{p}$ is the plane parameter. We find the cross-sectional tractness $A_{\alpha}(\beta, \gamma)$ by the intersection of the thresholded $2 \mathrm{D}$ coherence map and FA map computed based on $P_{Y(\alpha)}(\mathbf{p})$ and Equation (2):

$$
A_{\alpha}(\beta, \gamma)=\left\{C\left(P_{Y(\alpha)}(\mathbf{p}) ; Y(\alpha)\right) \geq \tau_{d p}\right\} \cap\left\{F_{f a}\left(P_{Y(\alpha)}(\mathbf{p})\right) \geq \tau_{f a}\right\}
$$

Specifically, the tract axis is $A_{\alpha}(\beta, 0)=Y(\alpha) \forall \beta$. The tract surface $A_{\alpha}(\beta, \pi)$ can be found by marching along the thresholded $2 \mathrm{D}$ coherence and FA map. 


\section{Step 1}

Seeding $L^{(0)}(s)$

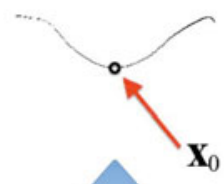

Step 2-3

$V_{\text {filtered }}^{(0)}(\alpha, \beta, \gamma)$
Step 4-5

Extended axis $L^{(1)}(s)$

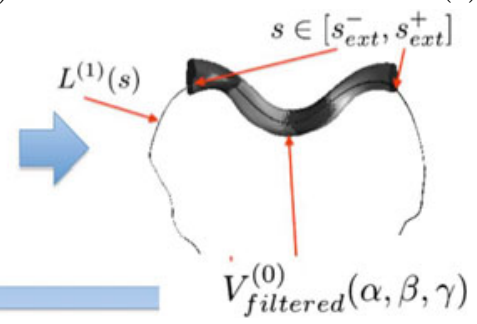

Fig. 2. The first iteration of the fasciculography algorithm in Section 2.2 for the reconstruction of the Tapetum portion of the corpus callosum from real DTI

\subsection{Tract Optimization}

Since all geometric entities are unknown at the beginning, the search is implemented in an iterative fashion. To find a maximum length tract-axis streamline $Y(\alpha)$ under the influence of the tract surface $S(\alpha, \beta)$ and a generic model $V(\alpha, \beta, \gamma)$ and vice versa, we first begin with a manually seeded streamline $L^{(0)}(s)$ anywhere within the tract. The superscript ${ }^{(t)}$ indicates the iteration number. There is a re-parametrization from $s$ to $\alpha$ since streamline tracking can move in both directions from the seed while we define $\alpha$ to be $[0, \pi]$. Since $s$ and $\alpha$ are a one-to-one mapping, we assume $s=s(\alpha)$ for simplicity. The steps of the algorithm are graphically illustrated in Figure 2 .

\section{Fasciculography (FASC) Algorithm}

Step 1: Given a seed point $\mathbf{x}_{0}$ on a vector field $\mathbf{F}$, we compute a streamline $L^{(0)}(s)$ using any numerical method solving Equation (1). Take this line to be the initial tract axis:

$$
V^{(0)}(\alpha, \beta, 0)=L^{(0)}(s) \quad \forall \beta
$$

Step 2: Compute the surface using Equation (3) and form the volume:

$$
V^{(0)}(\alpha, \beta, \pi)=S(\alpha, \beta)=A_{\alpha}(\beta, \pi)
$$

Step 3: Filter the volume with $\mathcal{P}_{\mathcal{K}}$ in the Fourier domain 17] removing frequencies higher than a tract length-dependent value $\mathcal{K}$ :

$$
V_{\text {filtered }}^{(0)}(\alpha, \beta, \gamma)=\mathcal{F}^{-1}\left\{\mathcal{P}_{\mathcal{K}} \mathcal{F}\left\{V^{(0)}(\alpha, \beta, \gamma\}\right\}\right.
$$

Step 4: Estimate the new tract axis :

$$
Y^{(0)}(\alpha)=\frac{\int_{\beta} V_{\text {filtered }}^{(0)}\left(\alpha, \beta_{i}, \pi\right) d \beta_{i}}{\int_{\beta} d \beta_{i}}
$$


Note that typically $Y^{(0)} \neq L^{(0)}$. The result of each iteration will be an improved axis that has taken tract smoothness and tensor coherence into account. However, it may be shorter than the optimum.

Step 5: Extend $Y^{(0)}(\alpha)$ to form $L^{(1)}(\alpha)$ by re-seeding and streamline tracking from the two end points of $Y^{(0)}(\alpha)$. Let $\left[s_{\text {ext }}^{-}, s_{\text {ext }}^{+}\right]$be the range of parameters indicating the portions of the new $L^{(1)}$ which is copied from Step 4 . We solve for $L^{(1)}(s)$ according to:

$$
\begin{aligned}
L^{(1)}(s) & =Y^{(0)}\left(\alpha_{1}\right) \quad \text { if } s \in\left[s_{\text {ext }}^{-}, s_{\text {ext }}^{+}\right] \\
\frac{d L^{(1)}(s)}{d s} & =\mathbf{F}\left(L^{(1)}(s)\right) \quad \text { otherwise }
\end{aligned}
$$

where re-parametrization of the new tract axis is $\alpha_{1}(s)=\pi\left(\frac{s-s_{e x t}^{-}}{s_{e x t}^{+}-s_{e x t}^{-}}\right)$.

Repeat Step 1 to 5 until no further extensions can be found, i.e. for some $t>0$, we stop iterating when $\left|L^{(t+1)}\right|=\left|Y^{(t)}\right|$. This algorithm always converges as it only allows increasing tract length and $\left\{\mathbf{x} \in \mathbb{R}^{3} \mid F_{f a}(\mathbf{x}) \geq \tau_{f a}\right\}$ is finite.

\section{Comparison Using Synthetic DTI Data}

We verify our method using a set of DTI synthetic phantom images with ground truth, PISTE [18]. Each tract in PISTE has a different geometry posing its own tracking challenge. We compare our method against FACT [4] in DtiStudio [8], TEND [5] in TrackVis [9] and Front Propagation [19|20]. The last spiral-cross tract is designed to reflect a more realistic situation where simple thresholding and shortest-path approaches in general would not trivially succeed. To summarize, we ran four methods on 12 synthetic DTIs ( 4 tract types, 3 noisy DTIs each) to get 48 tracking results. All methods use the mean FA value from the ground truth as the FA threshold and software default $0.5 \mathrm{~mm}$ for the step size. FACT/TEND use default $30^{\circ}$ as the maximum permissible turning angles.

\section{Qualitative Comparison (Phantoms)}

Figure 3 shows the 3D appearance of the tracking results using the tested methods in low Signal-to-Noise Ratio (SNR) environments. For the helix and spiralcross tracts, low or missing fiber coverage is observed between or distant from ROIs. Fibers are diverted outside the expected tract. This result shows the impact of premature termination of fibers due to accumulation of orientation errors. We demonstrated the intrinsic weakness of shortest-path approaches in [3(d). Tracks initiated far away from the source seed often diverted to adjacent tracts.

\section{Quantitative Comparison (Phantoms)}

Dice coefficients are computed for the different tract types in different SNR environments. A perfect segmentation will score 100. Figure 4 shows our method 

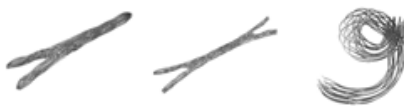

(a) Our method (FASC)
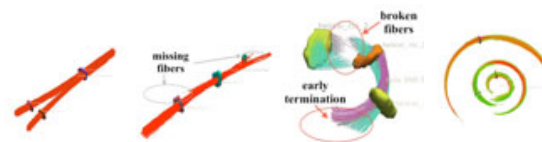

(c) Tensorline deflection (TEND)
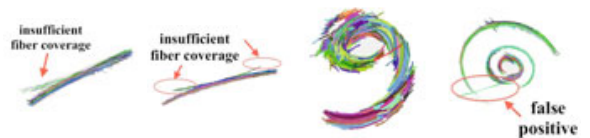

(b) $\mathrm{FACT}$
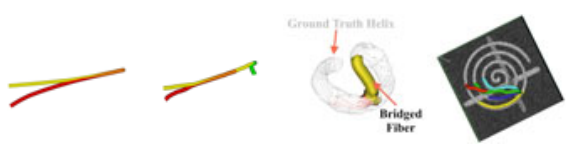

(d) Front-Propagation

Fig. 3. Comparing tracking methods in synthetic DTI with increasing tract difficulty (left to right) with SNR 5:1. ROIs for FACT/TEND are derived from ground truth. In a noisy environment, only FASC succeeds in all cases.
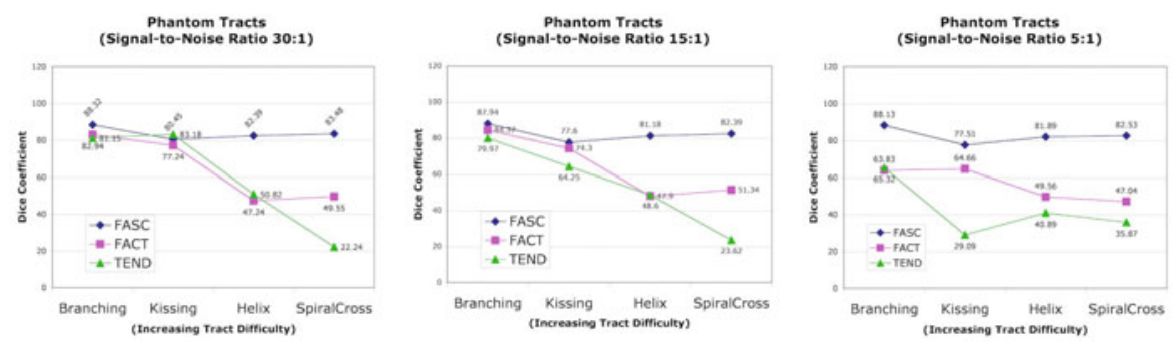

Fig. 4. Quantitative comparison of the tracking performance among Fasciculography (FASC), FACT and tensorline deflection (TEND) using synthetic DTI images. Each plot shows the tracking performance under the same Signal-to-Noise Ratio with increasing tract difficulty (data points from left to right).

has the highest score in 11 out of 12 cases. All FASC lines are relatively flat, as a function of tract difficulty, indicating the robustness of the method regarding difficult tract types, while the others exhibit steeper downward slopes. FASC lines are all above $75 \%$ showing our method is robust in noisy environments, while the other lines are much lower (below 70\%) at low SNR cases.

\section{Experiments Using Real DTI}

We acheive similar levels of accuracy (compared with Figure 4) using our FASC method on DTI of 18 cingulum tracts (in terms of averaged Dice coefficient) : 68.6 (FASC), 41.8 (FACT) and 57.1 (TEND). Figure 1(lower-left) shows fibers for more than 30 tracts from a human DTI. In Figure 5 , we show that our method is robust against a range of FASC parameters. Reasonable variation of the thresholds or seeding locations within a tract do not significantly reduce 

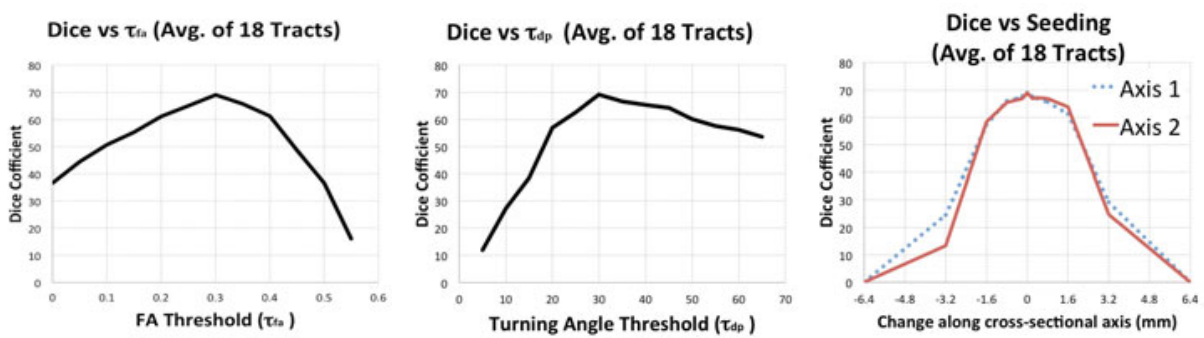

Fig. 5. Sensitivity to major FASC parameters. The Dice cofficients are computed based on 18 manual cingulum segmentations over FA slices.
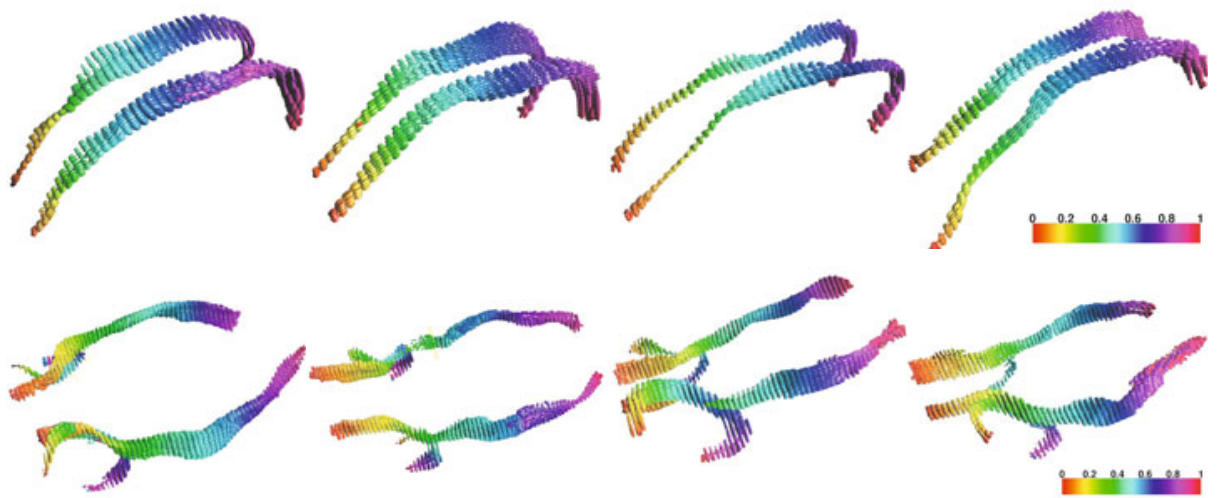

Fig. 6. Normalized cingulum (first row), inferior fronto-occipital and uncinate fasciculus voxels (second row) of 4 subjects from FASC. Voxels are colored by $\alpha$ parameter.

the accuracy of parcellation. Figure 6 shows the normalized tract voxels of the cingulum, inferior fronto-occipital (IFO) and uncinate (UNC) fasciculus. We choose the cingulum and IFO because they run approximately parallel to the sagittal and axial slices respectively which facilitates evaluation of the tracking results. On the other hand, cingulum and IFO bundles are challenging because they are long and thin. The UNC is relatively shorter but has higher curvature and appears to have lower FA ( 0.2 to 0.3 instead of $>0.4$ for cingulum and IFO) which makes it difficult to locate based on FA slices.

\section{Conclusion}

We have verified our method using third-party synthetic data [18] and real DTI.

We have packaged and extensively tested our interactive implementation in a 
standalone, open to the public application with a convenient user-interface 1 . The application can run on multiple OS platforms.

\section{References}

1. Basser, P.J., Mattiello, J., LeBihan, D.: Estimation of the effective self-diffusion tensor from the NMR spin echo. J. Magn. Reson B 103, 247-254 (1994)

2. Lori, N., Akbudak, E., Shimony, J., Cull, T., Snyder, A., Guillory, R., Conturo, T.: Diffusion tensor fiber tracking of human brain connectivity: aquisition methods, reliability analysis and biological results. NMR In Biomedicine 15, 493-515 (2002)

3. Alexander, A.L., Hasan, K.M., Lazar, M., Tsuruda, J.S., Parker, D.L.: Analysis of partial volume effects in diffusion-tensor MRI. Magn. Reson Med. 45, 770-780 (2001)

4. Mori, S., Crain, B.J., Chacko, V.P., van Zijl, P.C.: Three-dimensional tracking of axonal projections in the brain by magnetic resonance imaging. Ann. Neurol. 45, 265-269 (1999)

5. Lazar, M., Weinstein, D.M., Tsuruda, J.S., Hasan, K.M., Arfanakis, K., Meyerand, M.E., Badie, B., Rowley, H.A., Haughton, V., Field, A., Alexander, A.L.: White matter tractography using diffusion tensor deflection. Hum. Brain Mapp. 18, 306321 (2003)

6. Catani, M., Howard, R.J., Pajevic, S., Jones, D.K.: Virtual in vivo interactive dissection of white matter fasciculi in the human brain. Neuroimage 17, 77-94 (2002)

7. Faires, J.D., Burden, R.L.: Numerical methods, 3rd edn. Thomson/Brooks/Cole, Pacific Grove, CA (2003)

8. Jiang, H., van Zijl, P.C.M., Kim, J., Pearlson, G.D., Mori, S.: DtiStudio: resource program for diffusion tensor computation and fiber bundle tracking. Comput. Methods Programs Biomed. 81, 106-116 (2006)

9. Wang, R., Benner, T., Sorensen, A.G., Wedeen, V.J.: Diffusion toolkit: A software package for diffusion imaging data processing and tractography. Int'l Society of Magnetic Resonance in Medicine 15, 3720 (2007)

10. O'Donnell, L.J., Westin, C.-F.: Automatic tractography segmentation using a high-dimensional white matter atlas. IEEE Trans. Med. Imaging 26, 1562-1575 (2007)

11. Yushkevich, P.A., Zhang, H., Simon, T.J., Gee, J.C.: Structure-specific statistical mapping of white matter tracts. Neuroimage 41, 448-461 (2008)

12. O'Donnell, L.J., Westin, C.-F., Golby, A.J.: Tract-based morphometry for white matter group analysis. Neuroimage 45, 832-844 (2009)

13. Mohan, V., Sundaramoorthi, G., Tannenbaum, A.: Tubular surface segmentation for extracting anatomical structures from medical imagery. IEEE Trans. Med. Imaging 29, 1945-1958 (2010)

14. Smith, S.M., Jenkinson, M., Johansen-Berg, H., Rueckert, D., Nichols, T.E., Mackay, C.E., Watkins, K.E., Ciccarelli, O., Cader, M.Z., Matthews, P.M., Behrens, T.E.J.: Tract-based spatial statistics: voxelwise analysis of multi-subject diffusion data. Neuroimage 31, 1487-1505 (2006)

${ }^{1}$ Download @ http://www.fasciculography.com, http://noodle.med.yale. edu/ ho 
15. Corouge, I., Fletcher, P.T., Joshi, S., Gouttard, S., Gerig, G.: Fiber tract-oriented statistics for quantitative diffusion tensor MRI analysis. Med. Image Anal. 10, 786-798 (2006)

16. Ho, H.P., Wang, F., Blumberg, H.P., Staib, L.H.: Fast parametrized volumetric DTI tract parcellation. In: IEEE Int'l Symposium on Biomedical Imaging (2011)

17. Oppenheim, A.V., Willsky, A.S., Nawab, S.H.: Signals and systems, 2nd edn. Prentice-Hall, Upper Saddle River (1997)

18. Deoni, A.: Phantom Images for Simulating Tractography Errors, http://cubric.psych.cf.ac.uk/commondti

19. Jackowski, M., Kao, C., Qiu, M., Constable, R., Staib, L.: White matter tractography by anisotropic wavefront evolution and diffusion tensor imaging. Medical Image Analysis 9, 427-440 (2005)

20. Papademetris, X., Jackowski, M., Rajeevan, N., Okuda, H., Constable, R., Staib, L.: Bioimage Suite: An integrated medical image analysis suite, Section of Bioimaging Sciences, Dept. of Diagnostic Radiology, Yale School of Medicine 